\title{
Effect of peer evaluation training on senior nursing students' performance enrolled in nursing administration course
}

Reda A. Abo Gad, Heba K. Obied*

Faculty of Nursing, Tanta University, Tanta, Egypt

Received: June 14, 2016

DOI: $10.5430 /$ jnep.v7n4p55
Accepted: October 12, $2016 \quad$ Online Published: November 24, 2016

URL: http://dx.doi.org/10.5430/jnep.v7n4p55

\begin{abstract}
Objective: Senior nursing students have to be active participants in their learning process; this can be done through peer evaluation, hence they need to be trained to provide and accept constructive feedback to help their professional growth. So, this study aimed to assess the effect of peer evaluation training on senior nursing students' performance in nursing administration course.

Methods: The subject included all (152) available senior nursing students enrolled in nursing administration course at faculty of Nursing-Tanta University. Peer evaluation knowledge test (25 questions), nursing students' peer evaluation attitude scale (31 items) and nursing student's peer evaluation checklist (65 items) were used to collect the study data.

Results: Experimental nursing students group's total knowledge and performance about peer evaluation were significantly improved post than pre training sessions and than comparison nursing students group. Majority of experimental nursing students group agreed that peer evaluation was beneficial. Significant positive relation at $P \leq .05$ was found between the experimental and comparison nursing students groups' total level of knowledge, their attitude and peer evaluation performance post-sessions.

Conclusions: Senior nursing students' knowledge, performance and attitude about peer evaluation were improved after implementation of the training sessions. So, peer-evaluation method is recommended to be integrating into formal learning activities and establishing trustful reassuring learning environment.
\end{abstract}

Key Words: Peer evaluation, Senior nursing students, Nursing administration course, Training session

\section{INTRODUCTION}

Undergraduate education should equip nursing students with clinical as well as interpersonal skills to be able to assume their future role as competent professional nurses. ${ }^{[1]}$ Nursing students should take an active role in their learning process. Therefore, faculty educators should use different active learning strategies such as case studies, pair shares, role-playing, debate, cooperative learning and peer evaluation. ${ }^{[2,3]}$ Educators view peer evaluation strategy as an excellent tool to promote students' development, through which nursing stu- dents evaluate their colleagues' practice according to pre-set standards and give feedback to each other. ${ }^{[4,5]}$

Peer evaluation (PE) is defined as a strategy for providing feedback through which students significantly assess, evaluate, and judge the quality of their peers' behaviors and skills. ${ }^{[6]}$ Also, peer evaluation can be described as a teaching strategy that involves active participation of a student in the formative evaluation of another student's work as an evaluation technique, in which students appraise each other based on specific criteria. ${ }^{[7]}$

*Correspondence: Heba K. Obied; Email: hebaobied@yahoo.com; Address: Faculty of Nursing, Tanta University, Tanta, Egypt. 
Peer evaluation can be done individually or in groups. Using more than one evaluator is considered more effective and reliable. ${ }^{[3]}$ Peer evaluation can be applied for both formative as well as summative assessment. When peer evaluation is used in formative evaluations, it is very valuable and can both improve the learning experience and positively influence student personal and professional progress. ${ }^{[6,8]}$

Previous studies purported that peer evaluation helps students to recognize their strength as well as weak points, consider errors as a new opportunity for re-learning, and enhance their self-evaluation. Furthermore, peer evaluation enables nursing students to develop skills such as critical thinking, collaborative communication, negotiation, and critiquing skills. Utilization of peer evaluation strategy enhances learning process in many ways as: it integrates the evaluation into education process, relocates knowledge into practical skills, provides profound-learning experience, develops social interactions and trustful learning atmosphere and reinforces excellence. ${ }^{[9-11]}$

On the other hand, utilization of peer evaluation strategy confronted with a number of limitations including: students may not be competent or mature enough to provide accurate judgment, and some students may perceive it as an extra load. In addition some students may have negative feelings regarding peer evaluation as a result of potential for gaining negative feedback, difficulty in accepting feedback as an opportunity for growth rather than a personal attack. Also, students may feel uncomfortable as a reviewer, they may sound overly critical and judgmental when providing feedback for their peers. ${ }^{[4,5,12]}$

For effective application of peer evaluation, students need to identify who will evaluate them, what procedures will be included in the evaluation session, when the evaluation will be carried out, why to use peer evaluations, and how their peer evaluations will affect their own grades. ${ }^{[13]}$ Additionally, nursing students should be trained on how to use evaluation tools, learning objective have to be clear for them, and the faculty should create interdependence trustful environment. ${ }^{[14]}$ Moreover, to minimize the impact of friendship relations on peer evaluation process, the evaluator name as well as the student who is being evaluated should be hidden. ${ }^{[15]}$

Senior nursing students are those enrolled in the final undergraduate year at the baccalaureate nursing program, they need to posses different skills to be able to face real life situations, they successfully passed numerous courses as fundamental nursing, medical-surgical nursing, pediatric nursing, obstetric and gynecology nursing, psychiatric nursing, community health nursing, and nursing administration. Peer evaluation is a necessary skill for lifelong learning as well as professional development. Many studies encourage incorporate peer evaluation strategy as a formal evaluation method in higher education. Noteworthy, peer evaluation promotes higher levels of learning throughout meta-cognition process. ${ }^{[2,16,17]}$

\subsection{Aim of the study}

The current study aimed to assess the effect of peer evaluation training on senior nursing students' performance enrolled in nursing administration course.

\subsection{Research hypothesis}

Senior nursing students' peer performance in nursing administration course expected to be improved after implementing of peer evaluation training session.

\section{Materials \& Method}

\subsection{Design}

Non-equivalent pretest-posttest control group design was used.

\subsection{Setting}

The study was conducted at Nursing Administration Department, Faculty of Nursing-Tanta University- Egypt.

\subsection{Subject}

This study was carried out on all (152) available 4th year nursing students enrolled in nursing administration course during the first semester at their last academic year (2015). The study subject was divided into two identical groups; experimental nursing students group (76) and comparison nursing students group (76). They were assigned to 8 clinical areas in subgroups of around 10 students.

\subsection{Tools}

Three tools were used in the current study.

Tool I: Peer Evaluation Knowledge test developed by researchers after reviewing of related literatures, ${ }^{[18-20]}$ to assess nursing students' knowledge about peer evaluation. The tool consisted of two parts. Part 1: subject's data included gender, and previous year grade. Part 2: consisted of 25 questions in form of true \& false, and complete. It covered definition, benefits, process, principles and barriers to apply peer evaluation. Scorning: Subject's responses were scored by one for each correct answer and zero for incorrect answer. Total subject's knowledge levels classified into good $\geq 75$, fair $\geq 60-74$, and poor $<60$.

Tool II: Nursing Students' Peer Evaluation Attitude Scale developed by researchers guided by related literature. ${ }^{[21,22]}$ 
This tool consisted of 31 items to assess the subject's preference of peer evaluation (11 items), and attitude toward experiences gained from peer evaluation (20 items). Scorning: A five points Likert Scale was used, ranged from "strongly agree" to "strongly disagree". The responses were distributed into three categories: "strongly agree/agree" and "strongly disagree/disagree" and "neutral". The total responses ranged from 31 to 155 , the higher the scores indicating the stronger positive attitude regarding peer evaluation.

Tool III: Nursing Students' Peer Evaluation Checklist developed by researchers based on recent relevant literature ${ }^{[23,24]}$ to evaluate the nursing students' performance regarding applying different procedures taught in the practical areas of the nursing administration course. The tool represented two domains: First, professional behavior (14 items) including: communication (4 items), professionalism ( 7 items) and, teamwork (3 items). Second, nursing administration clinical duties (37 items) covered the following areas: Kardex, assignment sheet, team work sheet, on duty, off duty conference, shift report, problem solving sheet, time schedule and, safe administration of medication. Scorning: Each student evaluated his peer performances using 5-points scale ranging from 5 = almost always exhibits, $4=$ very often exhibits, 3 = often exhibits, 2 = occasionally exhibits and, $1=$ almost never exhibits.

\subsection{Procedures}

The study tools were reviewed by 7 experts in nursing administration from the Faculty of Nursing at Tanta University and their comments were considered and tools were modified. A pilot study was carried out on a sample of 15 nursing students; who were not included in the study subject; to ensure the clarity and applicability of the tools, identify any obstacles that may be encountered during data collection and, to estimate the time required for filling the questionnaire sheet. Reliability of the tools $(1,2,3)$ was tested using Cronbach's alpha coefficient test, its value was $0.75,0.79$ and 0.89 for tool $1,2,3$, respectively.

\subsection{Ethical considerations}

Official permission was obtained from nursing faculty responsible authorities. Afterwards, the purpose of the study was explained to the nursing students and informed consent to participate in the study was obtained from them. The subjects were assured that their data will be kept confidential, and their right to withdraw was assured.

\subsection{Field work}

(1) Assessment phase: Before starting the intervention, researchers carried-out pre-test to assess both experimental and comparison groups' baseline of knowledge and attitude about peer evaluation by using a written exam method at classroom using tools ( $1 \& 2$ ), the questionnaires collected immediately after completed. Researchers provided guidance about offering meaningful feedback and that peer evaluation would not affect students' overall grade to the clinical groups.

(2) Planning phase: Researchers designed the training session, specified the objectives, content and methods of teaching according to participants' need. Teaching methods included lecture and group discussion, researchers used different teaching aids including handouts, pen and paper and data show projector.

(3) Implementation phase: Researchers implemented a one-day workshop about peer evaluation strategy for the assistant teaching staff assigned to the clinical areas and the experimental group only. This workshop aimed at orientating the participants regarding peer evaluation basic definitions, aim and benefits, requirement, process, principles, clinical teaching staff roles and, barriers to apply peer evaluation method. Afterwards each student (at both groups) each other's clinical performance using tool (3) at clinical area.

- The experimental and comparison group students were assigned into eight sub-clinical areas. Researchers prepared a schedule for the trained students (experimental group) to act as peer evaluators for their colleagues (pre and post the training session). Each student in a group evaluated the performance of 3 or 4 of their peers and these scores were averaged for each student to minimize bias. The clinical sessions started from 9 AM to 1 PM two days/week for a month. The comparison group students evaluate each other performance only one time. The collection of data lasted two months. After finishing the study, the comparison group was subjected to the same workshop.

(4) Evaluation phase: By the end of the training session the nursing students' knowledge, attitude and, skills were re-assessed (immediate post-test) to evaluate the effect of the peer evaluation method using tools 1,2 , 3 . The students' results in experimental and comparison groups were compared pre and post the training sessions.

\subsection{Statistical analysis}

The collected data was organized, tabulated, and statistically analyzed using Microsoft Excel and Statistical Package for the Social Sciences (SPSS) version 20. For quantitative data the range, mean, and standard deviation were used. The dif- 
ference between two means was statistically analyzed using the student paired $(t)$ test. For qualitative data the number and percent distribution was calculated. Qualitative categorical variables were compared using chi-square test. The relationships between nursing student study and control group scores were assessed using Pearson's product-moment correlation coefficients. A $P$-value $<.05$ was considered statistically significant.

\section{RESUlts}

Table 1 shows the study subject's characteristic data. More than half (57.9\% and $53.9 \%)$ of nursing students at comparison and experimental nursing students groups were female. Equal percent $(21.1 \%)$ of both study groups had excellent grade, while, $(31.6 \%$ and $30.3 \%)$ of nursing students at comparison and experimental nursing students group had pass grade at the 3 rd academic year.

Table 1. The study subject's characteristic data

\begin{tabular}{|c|c|c|c|c|c|c|}
\hline \multirow{2}{*}{ Items } & \multicolumn{2}{|c|}{ Comparison group $(\mathrm{n}=76)$} & \multicolumn{2}{|c|}{ Experimental group $(n=76)$} & \multicolumn{2}{|c|}{ Total $(n=152)$} \\
\hline & No. & $\%$ & No. & $\%$ & No. & $\%$ \\
\hline \multicolumn{7}{|l|}{ Gender } \\
\hline Male & 32 & 42.1 & 35 & 46.1 & 67 & 44.1 \\
\hline Female & 44 & 57.9 & 41 & 53.9 & 85 & 55.9 \\
\hline \multicolumn{7}{|c|}{$3^{\text {rd }}$ year Grad } \\
\hline Excellent & 16 & 21.1 & 16 & 21.1 & 32 & 21.1 \\
\hline Very good & 16 & 21.1 & 18 & 23.7 & 34 & 22.4 \\
\hline Good & 20 & 26.3 & 19 & 25.0 & 39 & 25.7 \\
\hline Pass & 24 & 31.6 & 23 & 30.3 & 47 & 30.9 \\
\hline
\end{tabular}

Table 2 represents the experimental and comparison nursing students groups' total knowledge levels about peer evaluation pre and post training session. There was a statistical significant difference between both comparison and experimental nursing students groups' knowledge levels about peer evaluation at $(P<.05)$. More than half $(51.3 \%)$ of the experimental nursing students group total knowledge levels about peer evaluation method were poor pre session and decreased to $(19.7 \%)$ post session. Specifically, only $11.8 \%$ of experimental nursing students group had good level of knowledge pre session, which improved to $32.9 \%$ post session. Regarding to comparison nursing students group, pre session only $10.5 \%$ of comparison nursing students group had good level of knowledge about peer evaluation method and there was no statistical significant difference $(P<.05)$ post session.

Table 2. Distribution of the experimental and comparison nursing students groups' total knowledge levels about peer evaluation pre and post training session

\begin{tabular}{|c|c|c|c|c|c|}
\hline \multirow{2}{*}{ Knowledge levels about peer evaluation } & \multicolumn{2}{|c|}{ Comparison group $(n=76)$} & \multicolumn{2}{|c|}{ Experimental group $(n=76)$} & \multirow{2}{*}{$\begin{array}{l}\chi^{2} \\
P 1\end{array}$} \\
\hline & No. & $\%$ & No. & $\%$ & \\
\hline \multicolumn{6}{|l|}{ Pre session } \\
\hline Good & 8 & 10.5 & 9 & 11.8 & \multirow{3}{*}{$\begin{array}{l}0.13 \\
.934\end{array}$} \\
\hline Fair & 27 & 35.5 & 28 & 36.8 & \\
\hline Poor & 41 & 53.9 & 39 & 51.3 & \\
\hline \multicolumn{6}{|l|}{ Post session } \\
\hline Good & 11 & 14.5 & 25 & 32.9 & \multirow{3}{*}{$\begin{array}{l}14.85 \\
.0005 *\end{array}$} \\
\hline Fair & 29 & 38.2 & 36 & 47.4 & \\
\hline Poor & 36 & 47.4 & 15 & 19.7 & \\
\hline$\chi^{2}(P 2)$ & \multicolumn{2}{|c|}{$0.435(.647)$} & \multicolumn{2}{|c|}{$17.654(.0001 *)$} & \\
\hline
\end{tabular}

Note. $P 1$ comparison between pre and post in the same group; $P 2$ comparison between post in each group; ${ }^{*} P<.05$.

Table 3 displays mean and standard deviation of experimental and comparison nursing students groups' knowledge regarding peer evaluation sub-items at pre and post training session.
There was statistical significant improvement of experimental nursing students group mean scores in all items of peer evaluation knowledge post than pre session and than com- 
parison nursing students group's mean score at $(P \leq .05)$. the aim and benefits of peer evaluation item for comparison While, there was no difference of comparison nursing stu- and experimental nursing students groups, respectively, and dents group's mean scores in all items of peer evaluation knowledge post than pre session at $P<.05$. Pre program, the post program the experimental nursing students group mean score was improved to $4.09 \pm .89$.

highest mean score $(3.27 \pm 0.86$ and $3.21 \pm 0.95)$ was for

Table 3. Mean and standard deviation of experimental and comparison nursing students groups' knowledge regarding peer evaluation sub-items at pre and post training session

\begin{tabular}{|c|c|c|c|c|c|c|c|c|}
\hline \multirow{2}{*}{$\begin{array}{l}\text { Peer evaluation } \\
\text { Knowledge sub-items }\end{array}$} & \multirow{2}{*}{ Max } & \multicolumn{2}{|c|}{ Comparison group $(n=76)$} & \multirow{2}{*}{$\begin{array}{l}T \text {-test } \\
P 1\end{array}$} & \multicolumn{2}{|c|}{ Experimental group $(n=76)$} & \multirow{2}{*}{$\begin{array}{l}T \text {-test } \\
P 1\end{array}$} & \multirow{2}{*}{$\begin{array}{l}T \text {-test } \\
P 2\end{array}$} \\
\hline & & Pre & Post & & Pre & Post & & \\
\hline Definitions & 5 & $2.80 \pm 1.33$ & $2.84 \pm 1.41$ & $\begin{array}{l}0.56 \\
0.425\end{array}$ & $2.86 \pm 1.41$ & $4.22 \pm 0.79$ & $\begin{array}{l}2.97 \\
.013 *\end{array}$ & $\begin{array}{l}2.98 \\
.014 *\end{array}$ \\
\hline Aim \& benefit of PE & 5 & $3.27 \pm 0.86$ & $3.36 \pm 0.93$ & $\begin{array}{l}0.87 \\
0.365\end{array}$ & $3.21 \pm 0.95$ & $4.09 \pm 0.89$ & $\begin{array}{l}2.61 \\
.021 *\end{array}$ & $\begin{array}{l}2.65 \\
.026^{*}\end{array}$ \\
\hline Requirements for PE & 3 & $2.03 \pm 0.72$ & $2.13 \pm 0.84$ & $\begin{array}{l}0.99 \\
0.521\end{array}$ & $2.11 \pm 0.75$ & $2.55 \pm 0.57$ & $\begin{array}{l}1.98 \\
.036 *\end{array}$ & $\begin{array}{l}1.97 \\
.033^{*}\end{array}$ \\
\hline Principles of PE & 5 & $2.83 \pm 1.35$ & $2.94 \pm 1.41$ & $\begin{array}{l}0.98 \\
0.462\end{array}$ & $2.91 \pm 1.41$ & $4.25 \pm 0.83$ & $\begin{array}{l}2.99 \\
.011 *\end{array}$ & $\begin{array}{l}2.83 \\
.017^{*}\end{array}$ \\
\hline Process of PE & 5 & $3.11 \pm 1.16$ & $3.25 \pm 1.36$ & $\begin{array}{l}1.97 \\
0.365\end{array}$ & $3.06 \pm 1.14$ & $4.28 \pm 0.75$ & $\begin{array}{l}2.11 \\
.027 *\end{array}$ & $\begin{array}{l}2.21 \\
.028^{*}\end{array}$ \\
\hline Barriers for PE & 2 & $0.81 \pm 0.65$ & $0.85 \pm 0.46$ & $\begin{array}{l}0.65 \\
0.412\end{array}$ & $0.83 \pm 0.48$ & $1.82 \pm 0.42$ & $\begin{array}{l}4.52 \\
.001 *\end{array}$ & $\begin{array}{l}3.89 \\
.001 *\end{array}$ \\
\hline
\end{tabular}

Note. $P 1$ comparison between pre and post in the same group; $P 2$ comparison between post in each group; ${ }^{*}<.05$.

Table 4 demonstrates distribution of the experimental and comparison nursing students groups' total peer evaluation performance pre and post training session. There was statistically significant differences in the total peer evaluation performance between experimental and comparison nursing students groups $(P<.05)$. Overall post session, almost more than half $(55.2 \%)$ of experimental nursing students group had "always or very often exhibits" the peer evaluation performance, compared to $27.6 \%$ of those in the comparison nursing students group $(P<.0025)$.

Table 4. Distribution of the experimental and comparison nursing students groups' total peer evaluation performance pre and post training session

\begin{tabular}{|c|c|c|c|c|c|}
\hline \multirow{2}{*}{ Students' peer evaluation performance } & \multicolumn{2}{|c|}{ Comparison group $(n=76)$} & \multicolumn{2}{|c|}{ Experimental group $(n=76)$} & \multirow{2}{*}{$\begin{array}{l}\chi^{2} \\
P 1\end{array}$} \\
\hline & No. & $\%$ & No. & $\%$ & \\
\hline \multicolumn{6}{|l|}{ Pre session } \\
\hline Always or very often exhibits & 18 & 23.7 & 20 & 26.3 & \multirow{3}{*}{$\begin{array}{l}0.278 \\
.871\end{array}$} \\
\hline Often exhibits & 25 & 32.9 & 26 & 34.2 & \\
\hline Occasionally or almost never exhibits & 33 & 43.4 & 30 & 39.5 & \\
\hline \multicolumn{6}{|l|}{ Post session } \\
\hline Always or very often exhibits & 21 & 27.6 & 42 & 55.3 & \multirow{3}{*}{$\begin{array}{l}16.34 \\
.0001^{*}\end{array}$} \\
\hline Often exhibits & 26 & 34.2 & 24 & 31.6 & \\
\hline Occasionally or almost never exhibits & 29 & 38.2 & 10 & 13.2 & \\
\hline$\chi^{2}(P 2)$ & \multicolumn{2}{|c|}{$1.01(.132)$} & \multicolumn{2}{|c|}{$17.89(.0001 *)$} & \\
\hline
\end{tabular}

Note. $P 1$ comparison between pre and post in the same group; $P 2$ comparison between post in each group; ${ }^{*} P<.05$.

Table 5 displays mean and standard deviation of experimental and comparison group in response to each performance domain pre and post training session. The mean scores of experimental group were significantly higher than the scores graded by comparison group for all domains. Pre-session, experimental group provided their colleagues higher grades than they deserve in nursing administration clinical duties $(152.94 \pm 11.979)$ and professionalism $(25.26 \pm 3.186)$ domains than for the communication $(14.34 \pm 2.346)$ and teamwork $(10.29 \pm 2.008)$ domains, and these mean scores were improved post training session. 
Table 5. Mean and standard deviation of experimental and comparison groups in response to each performance domain pre and post training session

\begin{tabular}{|c|c|c|c|c|c|}
\hline \multirow{2}{*}{ Performance items } & \multirow{2}{*}{$\begin{array}{l}\text { Quest. } \\
\text { No }\end{array}$} & \multicolumn{2}{|c|}{ Experimental group $(\mathrm{n}=76)$} & \multicolumn{2}{|c|}{ Comparison group $(n=76)$} \\
\hline & & Mean \% & Mean \pm SD & Mean \% & Mean \pm SD \\
\hline \multicolumn{6}{|l|}{ Pre session } \\
\hline \multicolumn{6}{|l|}{ 1-Professional behavior } \\
\hline Communication & 4 & 71.7 & $14.34 \pm 2.346$ & 68.2 & $13.64 \pm 2.179$ \\
\hline Professionalism & 7 & 72.2 & $25.26 \pm 3.186$ & 67.9 & $23.78 \pm 3.238$ \\
\hline Teamwork & 3 & 68.6 & $10.29 \pm 2.008$ & 64.7 & $9.71 \pm 1.554$ \\
\hline \multicolumn{6}{|c|}{ 2-Nursing administration clinical duties } \\
\hline Kardex & 5 & 75.7 & $18.92 \pm 2.503$ & 71.2 & $17.81 \pm 2.435$ \\
\hline Assignment sheet & 5 & 72.1 & $18.04 \pm 2.822$ & 64.2 & $16.04 \pm 3.517$ \\
\hline Team work sheet & 5 & 77.0 & $19.24 \pm 2.693$ & 76.2 & $19.06 \pm 2.899$ \\
\hline On \& off duty conf & 5 & 70.4 & $17.61 \pm 3.154$ & 71.2 & $17.8 \pm 2.824$ \\
\hline Shift report & 5 & 69.9 & $17.49 \pm 2.992$ & 68.8 & $17.22 \pm 2.309$ \\
\hline Rota & 3 & 70.3 & $10.55 \pm 1.791$ & 70.3 & $10.55 \pm 1.519$ \\
\hline Problem solving sheet & 4 & 73.0 & $14.61 \pm 2.065$ & 71.4 & $14.29 \pm 2.289$ \\
\hline Medication safety & 5 & 70.4 & $17.59 \pm 3.156$ & 70.6 & $17.67 \pm 2.907$ \\
\hline Total & 37 & 82.7 & $152.94 \pm 11.979$ & 80.1 & $148.12 \pm 9.655$ \\
\hline \multicolumn{6}{|l|}{ Post session } \\
\hline \multicolumn{6}{|l|}{ 1-Professional behavior } \\
\hline Communication & 4 & 89.9 & $17.99 \pm 1.285$ & 68.7 & $13.74 \pm 2.201$ \\
\hline Professionalism & 7 & 92.3 & $32.29 \pm 2.341$ & 68.4 & $23.94 \pm 3.220$ \\
\hline Teamwork & 3 & 90.5 & $13.58 \pm 1.091$ & 66.0 & $9.90 \pm 1.592$ \\
\hline \multicolumn{6}{|c|}{ 2-Nursing administration clinical duties } \\
\hline Kardex & 5 & 89.3 & $22.32 \pm 1.770$ & 71.7 & $17.94 \pm 2.516$ \\
\hline Assignment sheet & 5 & 88.3 & $22.08 \pm 1.700$ & 64.9 & $16.24 \pm 3.466$ \\
\hline Team work sheet & 5 & 88.2 & $22.07 \pm 1.608$ & 76.9 & $19.24 \pm 2.895$ \\
\hline On \& off duty conf & 5 & 88.9 & $22.24 \pm 1.663$ & 75.9 & $18.99 \pm 2.748$ \\
\hline Shift report & 5 & 89.3 & $22.32 \pm 1.484$ & 69.8 & $17.45 \pm 2.324$ \\
\hline Rota & 3 & 89.8 & $13.47 \pm 0.884$ & 71.3 & $10.70 \pm 1.624$ \\
\hline Problem solving sheet & 4 & 88.6 & $17.71 \pm 1.402$ & 72.3 & $14.46 \pm 2.339$ \\
\hline Medication safety & 5 & 89.2 & $22.30 \pm 1.696$ & 71.5 & $17.87 \pm 3.040$ \\
\hline Total & 37 & 89.5 & $165.64 \pm 14.00$ & 80.3 & $148.66 \pm 12.435$ \\
\hline
\end{tabular}

Table 6 describes distribution of the experimental and comparison nursing students groups' total attitude about peer evaluation pre and post training session. As noticed in the table there was statistically significant change of experimental nursing students group's total attitude about peer evaluation method post than pre session and, than comparison nursing students group post session at $(P<.05)$. Pre session, considerable percent $(42.1 \%)$ of experimental nursing students group had negative attitude as a total about peer evaluation method, but, only $21.1 \%$ of them had positive attitude which significantly changed $(P<.05)$ post session and reached to one half $(50.0 \%)$ of them. According to comparison nursing students group, pre session, low percent $(19.7 \%)$ of them 60 had positive attitude as a total about peer evaluation, as well as, 40.8 of them had negative attitude, without significant difference post session at $(P<.05)$.

Agreement of experimental group nursing students upon experience gained from peer evaluation. Table 7 shows that the highest agreement was upon peer evaluation encourages transfer of learning and enhance the students learning experience (96\%), followed by developed skills for evaluating basic clinical skills (86.8\%) and, peer evaluation develops of student's judgment skills (85.5\%). On the other hand, $60.5 \%$ of experimental nursing students group had agreed that peer evaluation experience was time and effort spent. 
Table 6. Distribution of the experimental and comparison nursing students groups' total attitude about peer evaluation pre and post training session

\begin{tabular}{|c|c|c|c|c|c|}
\hline \multirow{2}{*}{$\begin{array}{l}\text { Students' attitude about peer } \\
\text { evaluation }\end{array}$} & \multicolumn{2}{|c|}{ Comparison group $(n=76)$} & \multicolumn{2}{|c|}{ Experimental group $(n=76)$} & \multirow{2}{*}{$\begin{array}{l}\chi^{2} \\
P 1\end{array}$} \\
\hline & No. & $\%$ & No. & $\%$ & \\
\hline \multicolumn{6}{|l|}{ Pre session } \\
\hline Positive attitude & 15 & 19.7 & 16 & 21.1 & \multirow{3}{*}{$\begin{array}{l}0.12 \\
.942\end{array}$} \\
\hline Neutral & 30 & 39.5 & 28 & 36.8 & \\
\hline Negative attitude & 31 & 40.8 & 32 & 42.1 & \\
\hline \multicolumn{6}{|l|}{ Post session } \\
\hline Positive attitude & 14 & 18.4 & 38 & 50.0 & \multirow{4}{*}{$\begin{array}{l}17.99 \\
.001^{*}\end{array}$} \\
\hline Neutral & 32 & 42.1 & 22 & 28.9 & \\
\hline Negative attitude & 30 & 39.5 & 16 & 21.1 & \\
\hline$\chi^{2}(P 2)$ & \multicolumn{2}{|c|}{$0.76(.231)$} & \multicolumn{2}{|c|}{$8.90\left(.001^{*}\right)$} & \\
\hline
\end{tabular}

Note. $P 1$ comparison between pre and post in the same group; $P 2$ comparison between post in each group; ${ }^{*} P<.05$.

Table 7. Agreement of experimental group nursing students upon experience gained from peer evaluation

\begin{tabular}{|c|c|c|c|}
\hline \multirow[b]{2}{*}{ Statements } & \multicolumn{3}{|c|}{ Experimental group $(\mathrm{N}=76)$} \\
\hline & $\begin{array}{l}\text { Agree } \\
\text { N (\%) }\end{array}$ & $\begin{array}{l}\text { Neutral } \\
\text { N (\%) }\end{array}$ & $\begin{array}{l}\text { Disagree } \\
\text { N (\%) }\end{array}$ \\
\hline The Experiencing peer evaluation was time and effort consuming* & $46(60.5)$ & $23(30.3)$ & $7(9.2)$ \\
\hline I now understand the principles behind evaluation & $64(84.2)$ & $8(10.5)$ & $4(5.3)$ \\
\hline I become skillful in evaluating basic clinical skills & $66(86.8)$ & $7(9.2)$ & $3(4.0)$ \\
\hline Experiencing peer evaluation helped me to reflect on my previous learning & $65(85.5)$ & $7(9.2)$ & $4(5.3)$ \\
\hline The curriculum should include more peer evaluation opportunities & $60(78.9)$ & $13(17.1)$ & $3(4.0)$ \\
\hline I enjoyed evaluating my peer students & $61(80.3)$ & $10(13.1)$ & $5(6.6)$ \\
\hline The peer evaluation experience was personally rewarding & $64(84.2)$ & $7(9.2)$ & $5(6.6)$ \\
\hline Peer evaluation increases student involvement and responsibility & $64(84.2)$ & $11(14.5)$ & $1(1.3)$ \\
\hline Peer evaluation develops of student’s judgment skills & $65(85.5)$ & $8(10.5)$ & $3(4.0)$ \\
\hline Peer evaluation encourages transfer of learning and enhance learning experience & $73(96.0)$ & $3(4.0)$ & $0(0.00)$ \\
\hline Peer evaluation allows students’ better understanding of assessment criteria & $64(84.2)$ & $7(9.2)$ & $5(6.6)$ \\
\hline Peer evaluation eliminates the ambiguity of the assessment process & $58(76.3)$ & $12(15.8)$ & $6(7.9)$ \\
\hline Peer evaluation facilitates learning from seeing others successes and weaknesses & $57(75.0)$ & $17(22.4)$ & $2(2.6)$ \\
\hline Peer evaluation increases student autonomy/independence & $61(80.3)$ & $13(17.1)$ & $2(2.6)$ \\
\hline Peer evaluation increases student confidence & $58(76.3)$ & $14(18.4)$ & $4(5.3)$ \\
\hline PE helps development of desirable attributes (negotiation, communication skills) & $57(75.0)$ & $17(22.4)$ & $2(2.6)$ \\
\hline Peer evaluation can develop students’ ability to work cooperatively & $63(82.9)$ & $8(10.5)$ & $5(6.6)$ \\
\hline Peer evaluation promotes lifelong learning skills & $61(80.3)$ & $12(15.8)$ & $3(4.0)$ \\
\hline Experiencing peer evaluation will help me to carryout my post graduate role & $62(81.6)$ & $13(17.1)$ & $1(1.3)$ \\
\hline Peer evaluation improves student motivation & $64(84.2)$ & $7(9.2)$ & $5(6.6)$ \\
\hline
\end{tabular}

* reverse items

Analysis of Table 8 verifies the effect of the knowledge levels about peer evaluation method on experimental nursing students group's total attitude and evaluation performance skills. Significant positive relation at $P \leq .05$ was found between experimental nursing students group's total level of knowledge and their attitude and evaluation performance regarding peer evaluation post session.

Table 9 reveals relation between experimental nursing students group's personal data and their knowledge, attitude and

Published by Sciedu Press

peer evaluation performance post training session. The table demonstrates statistically significant differences between experimental nursing students group gender and their knowledge grades, attitude and, performance scores about peer evaluation method $(P<.05)$. Significant positive relation at $(P<.05)$ was found between previous grades of experimental nursing students group and their knowledge and attitude about peer evaluation method, while, there was no statistical significant relation with their performance skills of peer evaluation method $(P<.068)$. 
Table 8. Relation between experimental group nursing students total knowledge level, their attitude and their peer evaluation performance about peer evaluation method post session $(\mathrm{N}=76)$

\begin{tabular}{|c|c|c|c|c|c|c|c|c|c|}
\hline \multirow{3}{*}{ Peer evaluation } & \multicolumn{6}{|c|}{$\begin{array}{l}\text { Knowledge level of experimental group about peer } \\
\text { evaluation, method post session }\end{array}$} & \multirow{2}{*}{\multicolumn{2}{|c|}{ Total }} & \multirow{3}{*}{$\begin{array}{l}\chi^{2} \\
p\end{array}$} \\
\hline & \multicolumn{2}{|c|}{ Good } & \multicolumn{2}{|c|}{ Fair } & \multicolumn{2}{|c|}{ Poor } & & & \\
\hline & No & $\%$ & No & $\%$ & No & $\%$ & No & $\%$ & \\
\hline \multicolumn{10}{|l|}{ Attitude about peer evaluation } \\
\hline Positive attitude & 20 & 80.0 & 16 & 44.4 & 2 & 13.3 & 38 & 50 & \multirow{3}{*}{$\begin{array}{l}16.58 \\
.0021^{*}\end{array}$} \\
\hline Neutral & 5 & 20.0 & 13 & 36.1 & 4 & 26.7 & 22 & 28.9 & \\
\hline Negative attitude & 0 & 0.0 & 7 & 19.4 & 9 & 60.0 & 16 & 21.1 & \\
\hline \multicolumn{10}{|l|}{ Peer evaluation performance skills } \\
\hline Always or very often exhibits & 21 & 84.0 & 18 & 50.0 & 3 & 20.0 & 42 & 55.3 & \\
\hline Often exhibits & 4 & 16.0 & 15 & 41.7 & 5 & 33.3 & 24 & 31.6 & 7.33 \\
\hline Occasionally or almost never exhibits & 0 & 0.0 & 3 & 8.3 & 7 & 46.7 & 10 & 13.2 & $.0158^{*}$ \\
\hline Total & 25 & & 36 & & 15 & & 76 & & \\
\hline
\end{tabular}

Table 9. Relation between experimental group's personal data and their knowledge, attitude and peer evaluation performance post training session $(\mathrm{N}=76)$

\begin{tabular}{|c|c|c|c|c|c|c|c|c|c|c|c|c|c|c|}
\hline \multirow{3}{*}{ Peer evaluation } & \multicolumn{4}{|c|}{ Gender } & \multirow{3}{*}{$\begin{array}{l}\chi^{2} \\
p\end{array}$} & \multicolumn{8}{|c|}{ Previous Grade } & \multirow{3}{*}{$\begin{array}{l}\chi^{2} \\
p\end{array}$} \\
\hline & \multicolumn{2}{|c|}{ Male } & \multicolumn{2}{|c|}{ Female } & & \multicolumn{2}{|c|}{ Excellent } & \multicolumn{2}{|c|}{ Very good } & \multicolumn{2}{|c|}{ Good } & \multicolumn{2}{|c|}{ Pass } & \\
\hline & No & $\%$ & No & $\%$ & & No & $\%$ & No & $\%$ & No & $\%$ & No & $\%$ & \\
\hline \multicolumn{15}{|c|}{ Knowledge about peer evaluation method } \\
\hline Good & 10 & 28.6 & 15 & 36.6 & \multirow{3}{*}{$\begin{array}{l}19.85 \\
.001^{*}\end{array}$} & 14 & 87.5 & 8 & 44.4 & 3 & 15.8 & 0 & 0.0 & \multirow{3}{*}{$\begin{array}{l}7.66 \\
.011^{*}\end{array}$} \\
\hline Fair & 15 & 42.9 & 21 & 51.2 & & 2 & 12.5 & 7 & 38.9 & 15 & 78.9 & 12 & 52.2 & \\
\hline Poor & 10 & 28.6 & 5 & 12.2 & & 0 & 0.0 & 3 & 16.7 & 1 & 5.3 & 11 & 47.8 & \\
\hline \multicolumn{15}{|l|}{ Attitude about peer evaluation method } \\
\hline Positive attitude & 16 & 45.7 & 22 & 53.7 & \multirow{3}{*}{$\begin{array}{l}5.02 \\
.021^{*}\end{array}$} & 12 & 75.0 & 10 & 55.6 & 8 & 42.1 & 8 & 34.8 & \multirow{3}{*}{$\begin{array}{l}4.42 \\
.017^{*}\end{array}$} \\
\hline Neutral & 15 & 42.9 & 7 & 17.1 & & 3 & 18.8 & 6 & 33.3 & 8 & 42.1 & 5 & 21.7 & \\
\hline Negative attitude & 4 & 11.4 & 12 & 29.3 & & 1 & 6.3 & 2 & 11.1 & 3 & 15.8 & 10 & 43.5 & \\
\hline \multicolumn{15}{|l|}{ Peer evaluation performance } \\
\hline Always/very often exhibit & 23 & 65.7 & 19 & 46.3 & \multirow{4}{*}{$\begin{array}{l}6.11 \\
.016^{*}\end{array}$} & 8 & 50.0 & 9 & 50.0 & 11 & 57.9 & 14 & 60.9 & \multirow{4}{*}{$\begin{array}{l}0.35 \\
.068\end{array}$} \\
\hline Often exhibits & 10 & 28.6 & 14 & 34.1 & & 6 & 37.5 & 6 & 33.3 & 6 & 31.6 & 6 & 26.1 & \\
\hline Occasionally/almost never exhibits & 2 & 5.7 & 8 & 19.5 & & 2 & 12.5 & 3 & 16.7 & 2 & 10.5 & 3 & 13.0 & \\
\hline Total & 35 & & 41 & & & 16 & & 18 & & 19 & & 23 & & \\
\hline
\end{tabular}

\section{Discussion}

Undergraduate nursing students should be actively involved in their learning process. Using peer evaluation strategy promotes nursing students' interdependence and socialization. ${ }^{[25]}$ Peer evaluation also enables nursing students to give and accept constructive criticism, and enhances students learning motives and refine their ability of self-evaluation, based on this formal peer evaluation must be incorporate into nursing education. ${ }^{[26]}$

\subsection{Nursing students' knowledge about peer evaluation}

Present study results showed that more than half of experimental group nursing students had poor levels of the total knowledge regarding peer evaluation pre sessions and this percent was decreased post session. In specific, minor percent of them had good knowledge level about peer evaluation method pre-session, which improved post session (see Table 2). Despite the increase, the scores remained low, perhaps this may indicate that one session was insufficient for some participants to learn the information presented. Also experimental group total knowledge levels post training sessions were better than those in comparison group. Attending peer evaluation training sessions enabled experimental group students to develop a better understanding of themselves as learners, encouraged to establish clear assessment criteria and assess their own and other students' achievement of task outcomes. 
Liu et al. (2010) ${ }^{[27]}$ and Fitzpatrick (2007) ${ }^{[28]}$ concluded that establishment of a strong knowledge base among participants, and their willingness to gain and share knowledge seriously affected the knowledge caliber. Along with the current study results, Triggs Nemshick et al. (1996) ${ }^{[29]}$ found that nursing students who received peer instruction had significantly higher cognitive test scores and moderately higher psychomotor test scores than those who received instruction from a clinical instructor. Karayurt et al. (2009) ${ }^{[30]}$ supported the present study results as they found that their study subjects' knowledge mean scores about breast cancer and breast self examination were similar between the two groups pre-intervention. While knowledge mean scores increased significantly six months post-intervention of peer and group education method.

Present study showed that pre program, the highest mean scores for comparison and experimental nursing groups were related to the aim and benefits of peer evaluation items, but post program the experimental group mean score was improved (see Table 3). In fact, the majority of those students agreed that they had many benefits from peer evaluation (see Table 7). This result indicated that those students did not experience peer evaluation before; and their participation in the training sessions were behind the elevation in their knowledge scores.

Peer evaluation empowers students to create knowledge that lead to enhance individual student's learning experience, therefore educators view peer evaluation strategy as a precious opportunity for students as it provide them valuable intuition into the evaluation process. ${ }^{[31]}$ Present study results were supported by Karayurt (2009) ${ }^{[30]}$ who found that both peer and group education resulted in an increase in perceived benefits. It is also expected that confidence should increase following education.

\subsection{Nursing students' peer evaluation performance}

The present study revealed that there was statistically significant differences in the nursing students' peer evaluation performance between experimental and comparison nursing students groups $(P<.05)$. Overall post session, almost more than half of experimental nursing group had "always or very often exhibits" the peer evaluation performance, compared to about one quarter of comparison group $(P<.0025)$ (see Table 4). Increased frequency of performing peer evaluation can be attributed to increase in knowledge and skills about peer evaluation method, increased perceived benefits and confidence, and decreased barriers. Peer assessment as an unbiased approach because group members have been working together for a period of time so they become knowledgeable about strengths and weakness of each other and

Published by Sciedu Press master the peer evaluation skills. Herbert (2007), ${ }^{[32]}$ Sadler and Good (2006) ${ }^{[33]}$ revealed that reactions on peer evaluation range from being viewed as 'fair and equitable' and 'qualified endorsements' to 'traditional peer assessment is relatively ineffective in addressing free-rider problems'.

Along with the present study results Karayurt et al. (2009), ${ }^{[30]}$ Tuna-Malak and Dicle (2007), ${ }^{[34]}$ Janda et al. $(2002)^{[35]}$ found that there were significant improvement rates of students performance regarding breast self examination following both peer and group education. Thus, the value of peer evaluation in undergraduate clinical nursing programs needs to be emphasized as it helps nursing students to be aware about their own strengths and weaknesses as compared with their peer and reinforces their learning objectives in clinical settings. ${ }^{[1]}$ Similarly, McDonald and Boud $(2003)^{[36]}$ reported that the skill of self assessment must be developed in high school students through training as it has a positive effect on the students achievement as well as overall performance.

Current result displayed that the mean scores of experimental nursing students group was significantly higher than those of comparison nursing students group for all domains of peer evaluation performance. Pre program, experimental nursing students group provided their colleagues higher grades than they deserve for the nursing administration clinical duties and professionalism domains than for the communication and teamwork domains, and these mean scores were improved post program and than comparison nursing students group (see Table 5). These results explained as clinical experiences enhanced students' progress; thus they gained the skills and confidence required to carry out their professional role. Additionally, students' interaction in clinical setting provides them with opportunity to closely observe different set of skills in their peers and critically evaluate their peers. These discrepancies between experimental and comparison nursing students groups suggested that when peer evaluation is used as part of the summative course assessment, students' ratings may be less reliable because it may falsely inflate the true academic value of student's performance.

The experimental nursing students' group scores differed least in the nursing administration clinical duties domain. This finding provides positive feedback to the faculty that students understand criteria and has similar expectations to that of faculty. Clinical practice increases student awareness of the need to develop their critical thinking skills. In addition, the Bachelor of Science in Nursing (BSN) program in which the students in this study participate has a strong emphasis on the utilization of the nursing administration clinical duties to develop critical thinking. 
Another major difference between the experimental and comparison nursing students groups evaluation scores was found in the domain of professionalism. Professional values and their associated behaviors are foundational to the practice of nursing. Peers can be viewed as vital source for learning, through role modeling and receiving feedback from colleagues; students become more open to accept constructive critique and follow professional behaviors. Finn et al. $(2010)^{[37]}$ and Speyer et al. (2011). ${ }^{[38]}$

\subsection{Nursing students' attitude of peer evaluation}

As noticed in Table 6, there was statistically significant improvement of experimental nursing students group students' total attitude about peer evaluation method post than pre sessions, and than comparison nursing students group at $P<.05$. This result might be due to during clinical rotations students interact with each other as a group they help each other and share the same clinical expectations. This interaction creates collaborative learning environment and enhances students' development of positive attitudes toward peers.

Previous researches concluded that receiving feedback from colleagues and clinical instructors enhanced nursing students' professional development and attitudes. They reported that nursing students feel more at ease when being observed and evaluated by their peers rather than by their clinical instructors. In addition, peer evaluation provides them with valuable insight into the assessment process and increases their self confidence. ${ }^{[39]}$ On the other hand, some students did not view receiving feedback from peer as a helpful strategy. ${ }^{[40]}$ Also, they viewed peer evaluation as being inadequate and lacking sufficient detail. ${ }^{[41]}$ Peer evaluation can be used in nursing clinical education, where nursing students constructively criticize their peers' communication, interviewing, problem solving skills, as well as other psychomotor skills. ${ }^{[42]}$

Current study result illustrates that the highest percentage of experimental nursing students' group agreement regarding the experiences gained from practicing peer evaluation included; peer evaluation encourages transfer of learning, enhances learning experience, and develops evaluation and judgment skills (see Table 7). This may be justified as those students enjoyed experiencing peer evaluation, they feel more relaxed and tension level from being evaluated by demonstrators decreased and their self confidence increased. Also, nursing students have improvements in their ability to selfidentify areas for improvement as a result of feedback received from their peers

Previous studies agreed with the present study results as Karayurt et al. (2009) ${ }^{[30]}$ who found that students peer review process was beneficial and helped. Also, Poon $(2011)^{[43]}$ found that majority of the students feel positively regarding peer assessment, and it was fair experience through which they gained new knowledge and skills. Meanwhile, Das et al. $(1998)^{[44]}$ mentioned that self and peer assessment positively affect students' motivation and self evaluation helps students to generally appraise their global performance.

Present study result illustrated that a considerable percent of experimental nursing students group agreed that peer evaluation experience was time and effort consuming (see Table 7). This may be due to the students' workload in preparing their assignments. The present study results was confirmed by Karayurt et al. (2009) ${ }^{[30]}$ who concluded that many factors mentioned by the students as causing dissatisfaction with the group peer review process were that it was time consuming.

Current study results demonstrated statistically significant differences between experimental group nursing students gender as well as previous grade and their knowledge grades, attitude and, performance scores about peer evaluation method $(P<.05)$ (see Table 9). This may be attributed to more than half of the subjects were female and they tend to be more organized, dutiful and persevering in their studies, also student previous grade suggested that the educational level strengthened the students' confidence and improved their performance of peer evaluation. Simsek and Balaban (2010); ${ }^{[45]}$ Kaenzig et al. (2006); ${ }^{[46]}$ and Meit et al. (2007) ${ }^{[47]}$ found in their studies that female students general performance appraisal is better than male undergraduate students as they are more strategic, tend to perform better individually than in teams and are more self-disciplined. On contrary, Langan et al. $(2008)^{[48]}$ found that there were no significant effects of gender of student on tutor grades.

\section{Conclusion}

Evaluating the efficacy of training session on senior nursing students' performance of peer evaluation method indicates that their knowledge, attitude and skills were generally poor pre training session. After implementation of the training, their knowledge scores significantly improved and their skills and attitude changed positively. This result suggests that there is potential for a greater applicability of this method of evaluation. Based on the findings of this study, we recommend that faculty administrators should integrate self-evaluation into formal learning activities, validate the criteria for peer evaluation to be clear and fully discuss with students, and ensure learning environment incorporates peer learning and evaluation in a range of ways. Also faculty educators should make student active participants in peer evaluation process, establish trustful and reassuring environment to prevent students' fear, train educators to master the peer evaluation strategies.

ISSN 1925-4040 E-ISSN 1925-4059 
Recommendations for future research on peer evaluation:

(1) The nature and extent of student involvement in peer evaluation.

(2) The quality of peer evaluation processes.

(3) Educators' perspective regarding their role in peer evaluation.

(4) The extent to which peer and self-evaluation are used in nursing faculty.

\section{ACKNOWLEDGements}

The researchers would like to thank College Dean and Head of Administration Department as they gave us the opportunity to complete the project. We also gratefully thank all the participants associated with the research.

\section{CONFlicts of InTEREST Disclosure}

The authors declare that there is no conflict of interest.

\section{REFERENCES}

[1] Youngshook Han, James DH, McLain RM. Relationships between student peer and faculty evaluations of clinical performance: A pilot study. Journal of Nursing Education and Practice. 2013; 3(8): 170-178. http://dx.doi.org/10.5430/jnep.v3n8p170

[2] Odom S, Glenn B, Sanner S, et al. Group Peer Review as an Active Learning Strategy in a Research Course. International Journal of Teaching and Learning in Higher Education. 2009; 21(1): 108-117.

[3] ŞAHIN S. An application of peer assessment in higher education. The Turkish Online Journal of Educational Technology-TOJET. 2008; 7(2).

[4] Boehm H, Bonnel W. The Use of Peer Review in Nursing Education and Clinical Practice. Journal For Nurses in Staff Development. 2010; 26 (3): 108-115. PMid:20508425 https : //doi.org/10.1097/NN D. 0 b013e3181993aa4

[5] Van den Berg I, Admiraal W, Pilot A. Design principles and outcomes of peer assessment in higher education. Studies in Higher Education. 2006; 31(3): 341-356. https : //doi .org/10 .1080/0307507060 0680836

[6] Topping KJ. Peer assessment. Theory Practice. 2009; 48: 20-27. https://doi.org/10.1080/00405840802577569

[7] Pond K, Ul-Haq R. Learning to assess students using peer review. Studies in Educational Evaluation. 1997; 23(4): 331-348. https ://doi.org/10.1016/S0191-491X (97)86214-1

[8] Black P, Harrison C, Lee C, et al. Working inside the black box.: Assessment for learning in the classroom. Phi Delta Kappan. 2004; 86(1): 13-22.

[9] Nicol DJ, Macfarlane-Dick D. Formative assessment and selfregulated learning: A model and seven principles of good feedback practice. Studies in Higher Education. 2006; 31(2): 199-218. https://doi.org/10.1080/03075070600572090

[10] Prins FJ, Sluijsmans D, Kirschner P, et al. Formative peer assessment in a CSCL environment: A case study. Assessment \& Evaluation in Higher Education. 2005; 40(4): 417-444. https : //doi .org/10.1 080/02602930500099219

[11] Johnson R. Peer assessments in physical education. Journal of Physical Education, Recreation \& Dance. 2004; 75(8): 33-41. https : //doi.org/10.1080/07303084.2004.10607287

[12] Magin D, Helmore P. Peer and teacher assessments of oral presentations: how reliable are they?. Studies in Higher Education. 2001; 26: 287-298. https://doi.org/10.1080/03075070120076264

[13] Gueldenzoph LE, May GL. Collaborative peer evaluation: Best practices for group member assessments. Business Communication Quarterly. 2002; 65(1): 9-20. https://doi.org/10.1177/10805699 0206500102

[14] Reese-Durham N. Peer evaluation as an active learning strategy. Journal of Instructional Psychology. 2005; 32(4): 338-348.
[15] Dochy F, Segers M, Sluijsmans D. The use of self-, peer and coassessment in higher education: a review. Studies in Higher Education. 1999; 24: 331-350. https ://doi .org/10.1080/03075079 912331379935

[16] Sluijsmans DM, Moerkerke G, Merrienboer JJ, et al. Peer assessment in problem based learning. Studies in Educational Evaluation. 2001; 27: 153-73. https://doi.org/10.1016/S0191-491X(01) 000 19-0

[17] Falchikov N, Goldfinch J. Student peer assessment in higher education: a meta-analysis comparing peer and teacher marks. Review of Educational Research. 2000; 70: 287-322. https ://doi .org/10 .3102/00346543070003287

[18] O’Conner AB. Clinical instruction and evaluation. 2nd ed. Boston, MA: Jones and Bartlett; 2006.

[19] Hernandez R. Benefits and Challenges of using self and peer assessment. UCD Teaching and Learning/ Resources. 2010.

[20] Pfeiffer JA, Wickline MA, Deetz J, et al. Assessing RN-to-RN peer review on clinical units. Journal of Nursing Management. 2012; 20(3): 390-400. PMid:22519617 https : //doi.org/10.1111/j . 1365-2834.2011.01321. $\mathrm{x}$

[21] El-Sayed SH, Metwally FG, Abdeen MA. Effect of peer teaching on the performance of undergraduate nursing students enrolled in nursing administration course. Journal of Nursing Education and Practice. 2013; 3(9): 156-166. http://dx.doi.org/10.5430/j nep.v3n9p156

[22] Spiller D. Assessment Matters: Self-Assessment and Peer Assessment. Teaching Developmentl Wahanga Whakapakari Ako, Waikato University. 2012.

[23] American Association of College of Nursing. The essentials of baccalaureate education for professional nursing practice. Available from: http://www.aacn.nche.edu/education-resources/B accEssentials08.pdf (31 October 2012, date last accessed).

[24] Nursing Administration Department. Clinical Practical Manual of Nursing Administration. Faculty of Nursing, Tanta University, Egypt. 2013.

[25] Mehrdad N, et al. Procedia-Social and Behavioral Sciences. 2012; 47: 1847-1852.

[26] Gerace L, Sibilano H. Preparing students for peer collaboration: A clinical teaching model. J Nurs Educ. 2006; 23: 206-209.

[27] Liu KL, Chang CC, Hu L. Exploring the effects of task characteristics on knowledge sharing in libraries. Library Review. 2010. 455-468 p.

[28] Fitzpatrick C. Training methods and tactical decision making simulations. Master's Thesis. Naval Postgraduate School. USA. 2007.

[29] Triggs Nemshick M, Shepard KF, Ladyshewsky R. Physical therapy clinical education in a $2: 1$ student-instructor education model. Phys Ther. 1996; 76: 968-984. PMid:8790275 
[30] Karayurt O, Dicle A, Tuna Malak A. Effects of Peer and Group Education on Knowledge, Beliefs and Breast Self-Examination Practice among University Students in Turkey. Turk J Med Sci. 2009; 39 (1): 59-66.

[31] Gagnon GW, Collay M. Designing for Learning: Six Elements in Constructivist Classrooms. Thousand Oaks, CA: Corwin Press; 2001

[32] Herbert N. Quantitative peer assessment: Can students be objective? In Proceedings of the 9th Australasian Computing Education Conference (ACE07). 2007. 63-71.

[33] Sadler PM, Good E. The impact of self- and peer-grading on student learning. Educational Assessment. 2006; 11: 1-31. https: //doi.org/10.1207/s15326977ea1101_1

[34] Tuna-Malak A, Dicle A. Assessing the efficacy of a peer education model in teaching breast self-examination to university students. Asian Pac J Cancer Prev. 2007; 8: 481-4. PMid:18260715

[35] Janda M, Stanek C, Newman B, et al. Impact of videotaped information on frequency and confidence of breast self-examination. Breast Cancer Res Treat. 2002; 73: 37-43. https ://doi.org/10.1023/ A : 1015264103561

[36] McDonald B, Boud D. The impact of self-assessment on achievement: The effects of self-assessment training on performance in external examination. Assessment in Education. 2003; 10(2): 209220. https://doi.org/10.1080/0969594032000121289

[37] Finn G, Garner J, Sawdon M. 'You're judged all the time!' Students' views on professionalism: A multicenter study. Med Educ. 2010; 44 814-825. PMid:20633221 http://dx.doi.org/10.1111/j.136 5-2923.2010.03743.x

[38] Speyer R, Pilz W, Van Der Kruis J, et al. Reliability and validity of student peer assessment in medical education: A systematic review. Med Teach. 2011; 33: 572-585. PMid:22022910 http://dx.doi.org/10.3109/0142159X.2011.610835

[39] Topping K. Peer assessment between students in colleges and universities. Rev Educ Res. 1998; 68: 249-276. https ://doi.org/10.3 102/00346543068003249
[40] Weidner TG, Popp JK. Peer assisted learning is effective in improving orthopedic evaluation psychomotor skills. J Athl Train. 2007; 42: 113-119. PMid:17597952

[41] Ladyshewsky R, Gotjamanos E. Communication skill development in health professional education: the use of standardized patients in combination with a peer assessment strategy. J Allied Health. 1997; 26: 177-186. PMid:9451588

[42] Arnold L, Shue CK, Kritt B, et al. Medical students' views on peer assessment of professionalism. J Gen Intern Med. 2005; 20: 819-824. PMid:16117749 https ://doi.org/10.1111/j.1525-1497.20 $05.0162 . \mathrm{x}$

[43] Poon J. Students' Perceptions of Peer Evaluation in Project Work. 2011. Australian Computer Society, Inc. This paper appeared at the 13th Australasian Computing Education Conference (ACE2011), Perth, Australia. Conferences in Research and Practice in Information Technology (CRPIT), Vol. 114. J. Hamer and M. de Raadt, Eds. Reproduction for academic, not-for-profit purposes permitted provided this text is included.

[44] Das M, Mpofu D, Dunn E, et al. Self and tutor evaluations in problembased learning tutorials: is there a relationship? Medical Education. 1998; 32(4): 411-418. PMid:9743805 https ://doi .org/10.104 6/j.1365-2923.1998.00217.x

[45] Simsek A, Balaban J. Learning Strategies of successful and unsuccessful university students. Contempory Educational Technology. 2010; 1(1): 36-45.

[46] Kaenzig R, Anderson S, Hyatt E, et al. Gender differences in students' perceptions of group learning experiences. Academy of Educational Leadership Journal. 2006; 10(1): 119-127.

[47] Meit S, Borges N, Cubic B, et al. Personality differences in incoming male and female medical students. Medical Education Online. 2007.

[48] Langan M, Shuker DM, Cullen WR, et al. Relationships between student characteristics and self-, peer and tutor evaluations of oral presentations. Assessment \& Evaluation in Higher Education. 2008; 33(2): 179-190. https : //doi .org/10.1080/02602930701292498 